\title{
MODÉLISATION D'UNE INTERACTION INDIVIDUS, ESPACE ET SOCIÉTÉ PAR LES SYSTÈMES MULTI-AGENTS : PÂTURE EN FORÊT VIRTUELLE
}

\author{
Jean-Luc Bonnefoy, François Bousquet, Juliette Rouchier
}

Belin | « L’Espace géographique »

2001/1 tome 30 | pages 13 à 25

ISSN 0046-2497

ISBN 2701129192

\section{Article disponible en ligne à l'adresse :}

https://www.cairn.info/revue-espace-geographique-2001-1-page-13.htm

Distribution électronique Cairn.info pour Belin.

(C) Belin. Tous droits réservés pour tous pays.

La reproduction ou représentation de cet article, notamment par photocopie, n'est autorisée que dans les limites des conditions générales d'utilisation du site ou, le cas échéant, des conditions générales de la licence souscrite par votre établissement. Toute autre reproduction ou représentation, en tout ou partie, sous quelque forme et de quelque manière que ce soit, est interdite sauf accord préalable et écrit de l'éditeur, en dehors des cas prévus par la législation en vigueur en France. Il est précisé que son stockage dans une base de données est également interdit. 


\section{Modélisation d'une interaction individus, espace et société par les systèmes multi-agents : pâture en forêt virtuelle}

\section{Jean-Luc Bonnefoy ${ }^{1}$, François Bousquet ${ }^{2}$, Juliette Rouchier ${ }^{3}$}

\begin{abstract}
RÉsumé.- Un système multi-agents permet de construire des modèles où les actions d'individus virtuels sont spatialisées et autonomes. Il est possible de mettre en place une interaction entre les représentations dynamiques que ces individus ou le groupe ont de l'espace et les contraintes que la collectivité leur impose. Dans cet exemple, nous avons simulé l'usage de la forêt par une communauté de bergers, chacun ayant un troupeau. Les résultats qui apparaissent au niveau global montrent qu'il existe une rétroaction très forte entre la manière de construire les représentations utilisées pour les choix d'actions - au niveau individuel ou collectif -, l'impact que celles-ci ont sur la structuration de l'espace et la transformation en retour des représentations elles-mêmes. Nous concluons sur le fait que cette technique de simulation se montre tout à fait adaptée à tester les hypothèses géographiques souhaitées.
\end{abstract}

ESPACE GÉOGRAPHIQUE, FORÊT, GESTION DURABLE, MODÉLISATION, REPRÉSENTATIONS SOCIALES, SYSTÈME MULTI-AGENTS

\begin{abstract}
Using multi-agent systems to model interaction between individuals, space and society: pasture in a virtual forest.- Multi-agent systems can be used to construct models in which the actions of virtual individuals are spatialised and autonomous. It is possible to model the interaction between the dynamic representations that the individuals or the group have of the space in question and the constraints that the community imposes on them. In this example, we have simulated the use of a forest by a community of shepherds, each of whom tends a flock. The results show strong retroaction between the construction of the individual or collective representations that underpin choices of actions, the impact that these representations have on the way the space is structured, and the subsequent transformation of the representations. We conclude that this simulation technique is particularly well suited to testing geographical hypotheses.
\end{abstract}

FOREST, GEOGRAPHICAL SPACE, MODELLING, MULTI-AGENT SYSTEM, SOCIAL REPRESENTATIONS, SUSTAINABLE MANAGEMENT
1. UMR 6012 «ESPACE », Université de Provence, 29 rue Robert Schumann, 13621 Aix-en-Provence.

2. «Gestion des ressources renouvelables », CIRAD-GREEN, BP 5035, 34090 Montpellier.

3. Centre of Policy Modelling, Manchester Metropolitan University, Manchester M13GH, UK.

\section{Introduction}

Les géographes ont élaboré des théories expliquant des processus spatiaux, par exemple pour sous-tendre la structuration des villes en un système hiérarchisé. Ils ont également mis en actes ces théories grâce à de nombreuses modélisations qui s'appliquent à décrire les formes d'organisation du système urbain et les flux qui les animent (Wilson, 
1970 ; Haynes, Fotheringham, 1988 ; Cattan, Grasland, 1994). Il existe aussi une profusion d'outils pour décrire et enrichir la compréhension des formes spatiales. Par exemple, l'étude des tissus urbains par l'analyse fractale tente de mettre en évidence des principes d'auto-organisation qui accompagneraient la croissance des morphologies irrégulières que sont les agglomérations urbaines (Frankhauser, 1994). Mais il existe peu de modélisations pour essayer de reproduire une forme à partir de sa genèse supposée. Nous tentons, dans une recherche dont nous verrons ici un premier pas, d'élaborer une modélisation de ce type.

Les travaux qui correspondent particulièrement à cette démarche sont de deux types. Dans le premier cas, il s'agit de construire des systèmes dynamiques à base d'équations différentielles et de descripteurs macroscopiques des systèmes étudiés pour s'intéresser à l'articulation des interactions au niveau microscopique, par exemple pour simuler la dynamique d'un semis urbain dans son organisation spatiale et hiérarchique (Allen, Sanglier, 1981 : travaux ayant pour fondement les structures dissipatives d'Ilya Prigogine; Pumain et al., 1989; Sanders, 1992 : travaux insistant sur le caractère foncièrement auto-reproducteur du système urbain).

Dans le second cas, la démarche s'inverse et prend un point de vue local. Il s'agit d'utiliser des règles au niveau microscopique pour simuler la mise en place et la dynamique de structures spatiales à l'échelle macroscopique. Cette approche prend plus facilement en compte la spatialité que la démarche ci-dessus à base d'équations différentielles. Le «jeu de la vie» en est un exemple très connu. Imaginé en 1970 par John Conway (1970), cet univers imaginaire en deux dimensions est constitué d'une matrice carrée composée de cellules munies de règles les plus simples possibles qui commandent la vie et la mort des cellules. En fonction de ces règles (une composition logique du contenu des cellules voisines), des structures périodiques émergent au niveau macroscopique et évoluent dans cet univers virtuel. Elles sont dotées de propriétés absentes au niveau microscopique et qu'il est impossible de déduire des règles établies entre cellules. Ce type de modélisation a été formalisé sous l'expression d'automates cellulaires (travaux de John von Neumann dans les années 1950). Ces «réseaux booléens [permettent] d'étudier sur des exemples relativement simples un problème très difficile mais qu'on rencontre souvent, celui du passage d'un niveau à l'autre, de l'élémentaire au général, du local au global» (Atlan, 1981). Dans le concert des travaux sur la vie et l'intelligence artificielles, les systèmes multi-agents (SMA), tout en conservant les mêmes fondements que les automates cellulaires, permettent notamment d'établir des relations spatiales plus complexes que la contiguiité ainsi que des relations non explicitement spatiales.

Les pages qui suivent s'insèrent dans des recherches entreprises distinctement par les auteurs pour la modélisation de dynamiques spatiales ou sociales, et qui trouvent ici une voie méthodologique commune. François Bousquet s'intéresse à la modélisation des interactions entre des dynamiques naturelles et sociales par les SMA dans le cadre de recherches sur la gestion des ressources renouvelables ${ }^{4}$. Dans ce même cadre, Juliette Rouchier s'attache à l'étude des relations d'échanges entre individus dans une société et principalement au rôle de la confiance. Jean-Luc Bonnefoy s'intéresse à la construction de dynamiques entre pratiques, représentations et structures spatiales qui produisent un territoire ou qui sont agies par lui. Ce texte souhaite participer au renouvellement d'un regard sur le territoire, tant le développement des recherches à son propos s'est tendu vers une théorie sociale parfois oublieuse de ses sources et contraintes spatiales; et vice-versa! Il s'intègre dans un programme des UMR 5603 et 6012 dont les motivations sont de faire dialoguer analyse spatiale et géographie sociale.

\section{Systèmes multi-agents et espace géographique}

L'intelligence artificielle à ses débuts entendait essentiellement rivaliser avec l'être humain. Il s'agissait de résoudre une question posée en adoptant le comportement et le savoir-faire d'un ou souvent plusieurs experts, d'où les programmes informatiques biens connus appelés «systèmes experts », notamment pour le diagnostic médical. Cette voie de l'intelligence artificielle soulève, entre autres questions, le problème de l'individualisation des compétences

4. L'application mise en œuvre ici a bénéficié des avancées de la plate-forme CORMAS (Common Pool Resources for Multi-Agent System), mise au point au CIRAD. 
d'expert dans un seul programme informatique. En effet, dans la plupart des cas pratiques, les savoirs nécessaires à la résolution de tâches complexes sont souvent distribués entre plusieurs individus. Ce sont des échanges d'informations, de connaissances spécifiques voire des négociations qui concourent à la résolution d'une tâche ou à l'établissement d'un diagnostic. Aussi, un courant de l'intelligence artificielle s'est inscrit dans la construction de programmes informatiques relativement autonomes et indépendants en interaction, appelés des «agents ». Un SMA est un programme informatique regroupant des petits programmes disposant d'une certaine autonomie constituant des entités artificielles qui évoluent, communiquent et agissent dans un environnement, qui n'est pas forcément spatial. Un SMA intègre une dimension temporelle sous la forme d'une horloge qui rythme les interactions entre ces diverses entités artificielles ou agents. Ces interactions produisent des organisations qui, en retour, sont susceptibles de contraindre les agents.

Selon Jacques Ferber (1995), un agent, dans une définition strictement informatique, est une «entité capable d'agir sur elle-même et sur son environnement, qui réagit à ses transformations et qui possède une représentation partielle de cet environnement». Les SMA, parce qu'ils donnent «la possibilité de modéliser directement les individus, leurs comportements et leurs interactions, offrent des solutions radicalement nouvelles pour la modélisation». Par exemple, le fonctionnement d'une fourmilière peut être modélisé en donnant aux fourmis des statuts d'agents dont les caractéristiques varieront avec les différentes spécialisations de ces insectes au sein de la société des fourmis (Drogoul et al., 1993). Par extension, le statut d'agent est également donné à des entités moins «actives» comme les ressources (nourriture, humidité). En évoluant dans un espace modélisé sous la forme de grilles régulières où sont réparties des ressources (voire une reconstitution plus complexe d'une réalité observée), chaque agent mobile se situe dans l'espace et par son action le transforme pour les autres. Les interactions sont au centre de ce type de modélisation.

Ces propriétés peuvent être mises à profit en géographie. En effet, «la modélisation multi-agents repose sur la capacité des programmes actuels à pouvoir donner une autonomie de comportement à des individus » (les agents) qu'ils soient des personnes, des animaux mais aussi des arbres, de la nourriture, etc. ou plus englobants comme un troupeau, ou encore des entités territoriales comme un village, une ville. Une approche géographique des SMA a modélisé l'évolution d'un système de villes sur une longue période de temps (Bura et al., 1996), et notamment sa hiérarchisation en termes de population et de fonctions urbaines. La croissance des villes s'opérait en conformité avec les théories sur les hiérarchies urbaines, la spécialisation des activités s'effectuant grâce à des mécanismes d'offre et de demande. Là, les concepteurs ont travaillé à un certain niveau d'abstraction : les entités élémentaires sont des lieux de peuplement, délaissant l'étude du comportement des acteurs individuels au profit des interactions entre agents «villes», ceci afin de retrouver des propriétés macroscopiques du système de villes.

Dans le cadre d'un univers multi-agents, imaginons que les sphères spatiale, individuelle et sociale s'articulent selon la figure 1, même si cette formalisation sous la forme de trois cercles distincts est caricaturale. Elle permettra cependant d'identifier clairement des types de modélisation multiagents. Les trois interactions doubles donnent lieu à des types différents de modélisation. L'interaction individusociété trouve son expression informatique dans la conception d'un agent «groupe social» qui va régler (contraindre ou permettre) certaines pratiques d'agents «individus» qui sont rattachés à ce groupe. Il existe deux manières de réaliser une mosaïque sociale au sein des SMA: soit elle est définie a priori par le concepteur lors de la construction du modèle, soit le modèle intègre un processus de différenciation sociale grâce à une division du travail parmi des

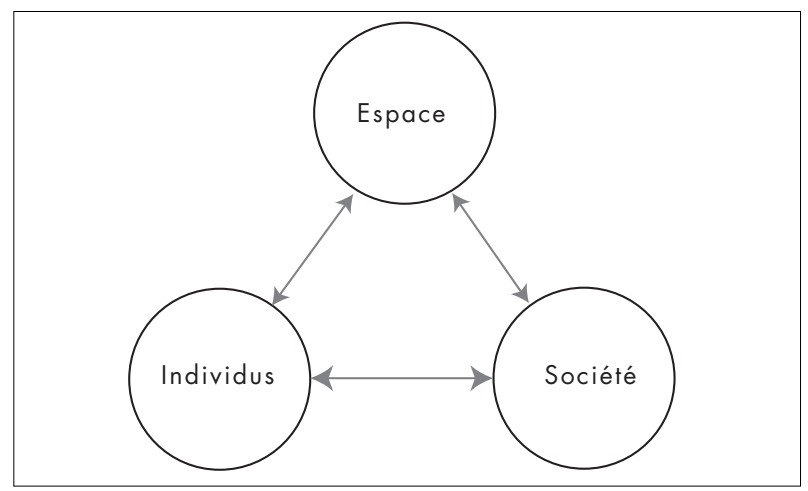

Fig. 1.-Une simulation au cœur de l'interaction individu, espace et société. 
«agents » initialement identiques. Une telle sociogenèse a été éprouvée avec succès par le modèle MANTA simulant une fourmilière (Drogoul et al., 1993). Ce modèle intégrait également la sphère spatiale sous la forme d'une interaction individu-espace.

L'intersection individu-espace constitue un des grands intérêts des SMA. Elle est traduite par les interactions spatiales qui se produisent entre un agent «individus» et un agent «ressource» ou entre agents «individus», en des lieux de l'univers multi-agents. Ces interactions qui se produisent dans le local peuvent être de concurrence ou de coopération pour atteindre une ressource. Beaucoup d'applications multi-agents sont de ce type quelles que soient les disciplines scientifiques qui les utilisent. Elles exploitent la capacité de l'espace à diversifier les contraintes selon les lieux, c'est-à-dire à s'extraire des modèles écologiques pour créer une interaction spatiale.

La modélisation de l'interaction espace-société est assez caractéristique des géographes. Elle est le contrepoids de la précédente attitude pour explorer la sphère spatiale et confère à des structures spatiales ou à des entités territoriales le statut d'agent. Les travaux évoqués plus haut concernant l'évolution d'un système de villes sont en partie de ce type.

Un autre travail (Bousquet, Gautier, 1999) met en scène les deux cas évoqués précédemment sur l'exemple d'un processus de conquête agricole : des paysans cultivent des terres fertiles à l'entour de leur village dont la population s'accroît et des bêtes parcourent librement l'espace, participant à une dégradation plus ou moins rapide de la forêt en savane. Dans une première modélisation, l'espace est support des ressources pour les troupeaux (la forêt) et les paysans (des lieux fertiles). Les agents ont des règles de comportements spatiaux, ils modifient leur environnement et perçoivent de manière objective toutes les modifications dans leur champ de perception. Dans une seconde modélisation, trois entités spatiales de type forêt, champs, savane sont établies a priori. Les auteurs mettent ainsi en avant la structuration spatiale de cet univers multi-agents. Ces ensembles spatiaux sont munis de caractéristiques d'agents alors que troupeaux et paysans n'apparaissent plus qu'à l'état de règles de fonctionnement de ces entités. Ces deux approches constituaient, pour les auteurs, le début d'une réflexion sur l'intégration de l'espace dans les SMA. Elles peuvent être approfondies car, dans le premier cas, il n'y a pas de modélisation de ces autres types de contingences, à savoir le poids du social sur les comportements, le poids des représentations collectives et individuelles de l'espace. Par exemple, la représentation collective d'un état de la forêt qui se dégrade au cours de la simulation ne peut être réintroduite dans la simulation. Dans le deuxième cas, où les agents sont des ensembles spatiaux, la modélisation suppose leur existence a priori. Outre que la modélisation d'échanges entre entités spatiales peut paraître peu naturelle, on pourrait imaginer que les comportements individuels donnent éventuellement lieu à des structures spatiales identifiables par les agents en cours de simulation, ce qui ne peut être le cas ici.

Nous avons envisagé une modélisation qui relève des trois sphères individuelle, spatiale et sociale. C'est une simulation du fonctionnement d'un espace géographique tel qu'il est généralement défini dans le champ de la géographie : "Acteurs individuels, groupes et institutions créent l'espace par leur travail, par leurs actes quotidiens, même ceux qui ne portent pas directement sur l'espace. Ils le font sur un terrain déjà défini, muni de "mémoires", qu'ils utilisent ou qu'ils transforment selon leurs moyens et leurs stratégies, lesquelles sont guidées en particulier par leurs représentations de l'espace lui-même. Par ses propres structures, l'espace qu'ils produisent les amène à infléchir leurs actions ultérieures, selon ces représentations, également modifiées par les nouvelles pratiques de l'espace transformé» (Brunet et al., 1992).

Il s'agit de construire, telle qu'elle est évoquée dans la dernière phrase de la définition ci-dessus, une dialectique entre un espace produit par la société et un espace contraignant les individus, notamment par le biais des représentations individuelles et collectives. Les représentations agissent comme médiateur dans la modélisation des interactions entre les agents et la ressource commune (Bousquet et al., à paraitre). Ici cependant, les représentations sont des constructions individuelles ou collectives issues de pratiques de l'espace, les unes et les autres évoluant au cours de la simulation au lieu d'être des référents communs établis a priori comme, par exemple, des pratiques religieuses (Lansing, Kremer, 1994). Cette démarche est plus qu'une intégration de l'espace dans les systèmes multi-agents, c'est la mise en scène de conceptions théoriques de l'espace géographique. Ainsi, dans le 
modèle, nous considérons trois éléments en interaction : l'espace en ses lieux et en ses structures à méso-échelle, les pratiques spatiales individuelles, ainsi que des appréciations de l'espace par les individus puis par le groupe. Dans la dynamique qui s'ensuit, les décisions du groupe affecteront les pratiques spatiales individuelles. L'espace produit sous contrainte exercera à son tour une contrainte sur les individus et la société par l'intermédiaire de ses nouvelles configurations. Ces différentes phases interagissent en un cycle temporel. La dynamique du modèle viendra en partie de ce décalage temporel entre les phases d'action, de représentation et de décision, en partie du décalage entre aspects microscopiques et macroscopiques.

Il apparaît intéressant d'articuler dans une même modélisation des interactions entre deux niveaux d'analyse pour la société et l'espace : le niveau des acteurs individuels et le niveau collectif d'une part; le niveau d'entités spatiales locales (les lieux ou cellules dans le modèle) et celui d'entités spatiales construites à méso-échelle (les structures spatiales composées de regroupements de cellules dans le modèle) d'autre part. Au cours du temps, ces deux niveaux se de manière dynamique grâce à une articulation entre individus, espace et société. Au total, notre recherche à venir s'attachera à déterminer :

- dans quelle mesure des règles définies au niveau individuel ou au niveau collectif sont susceptibles d'une réification dans l'espace de cet univers multi-agents?

- quel est l'effet de formes spatiales sur des pratiques individuelles et des décisions collectives?

- quels sont les niveaux de représentation de l'espace nécessaires et suffisants pour faire émerger certaines structurations de l'espace?

Une modélisation prenant en compte les niveaux local et global est pertinente pour deux raisons. Tout d'abord, il est difficile de simuler des interactions à l'échelle de groupes ou à méso-échelle (en ce qui concerne l'espace), sans analyser les interactions à l'intérieur de ces groupes ou aires puisque ces derniers participent à la cohésion et à la redéfinition dynamique des groupes ou entités spatiales. Ensuite, à l'opposé de la modélisation de l'évolution d'un système de villes (Bura et al., 1996), les règles à méso-échelle ne sont pas forcément connues ni théorisées. Compte tenu de la somme de travaux effectués sur le système de villes ainsi que de leurs objectifs, les auteurs pouvaient facilement s'affranchir des interactions au niveau des acteurs individuels et faire l'hypothèse simplificatrice de prendre les lieux de peuplement comme unité élémentaire du modèle. En d'autres cas, ce report de la complexité de l'échelle locale à la méso-échelle n'est pas possible ou souhaitable, notamment quand les entités spatiales définies à mésoéchelle (les structures spatiales) se déforment ou se reconstituent de manière dynamique sous l'action de divers agents et qu'il est primordial que ces recompositions affectent à nouveau les pratiques des agents.

Cette modélisation est entreprise afin de dégager l'intérêt des systèmes multi-agents pour l'appréhension d'un espace géographique et, de manière plus générale, s'intéresser à la dynamique entre représentations individuelles et collectives, ainsi qu'à la réification de cette dynamique et les contraintes en retour que cette dernière impose.

\section{Description du modèle}

Le modèle décrit des interactions entre le parcours de troupeaux conduits par des bergers et une ressource forestière, par le truchement de représentations spatiales. La pâture sera plus ou moins réglementée de manière dynamique par les représentations individuelles ou collectives qui se construisent au cours de la simulation, intégrant les états successifs de la ressource forestière entre altération en savane et repousse.

Procédons en suivant le cheminement de l'architecture du modèle (fig. 2). Au départ, 40 agents «bergers et troupeaux » sont préalablement placés au hasard dans un espace tel qu'il est présenté figure 3. Cet espace virtuel est le même pour toutes les simulations entreprises et les agents «bergers et troupeaux» seront placés en ce même lieu à chaque simulation. Ils constituent 40 troupeaux de taille identique guidés par leurs bergers qui vont se déplacer de manière aléatoire de cellule en cellule, l'un après l'autre à chaque pas de temps. Ces déplacements séquentiels sont caractéristiques des SMA asynchrones en opposition aux SMA synchrones où tous les agents «bergers et troupeaux» se déplaceraient en même temps. Cette éventualité impliquerait de gérer des priorités de pâture entre agents, ce qui n'est pas l'objet de notre modèle. Ici la règle est : «premier arrivé, premier servi». 


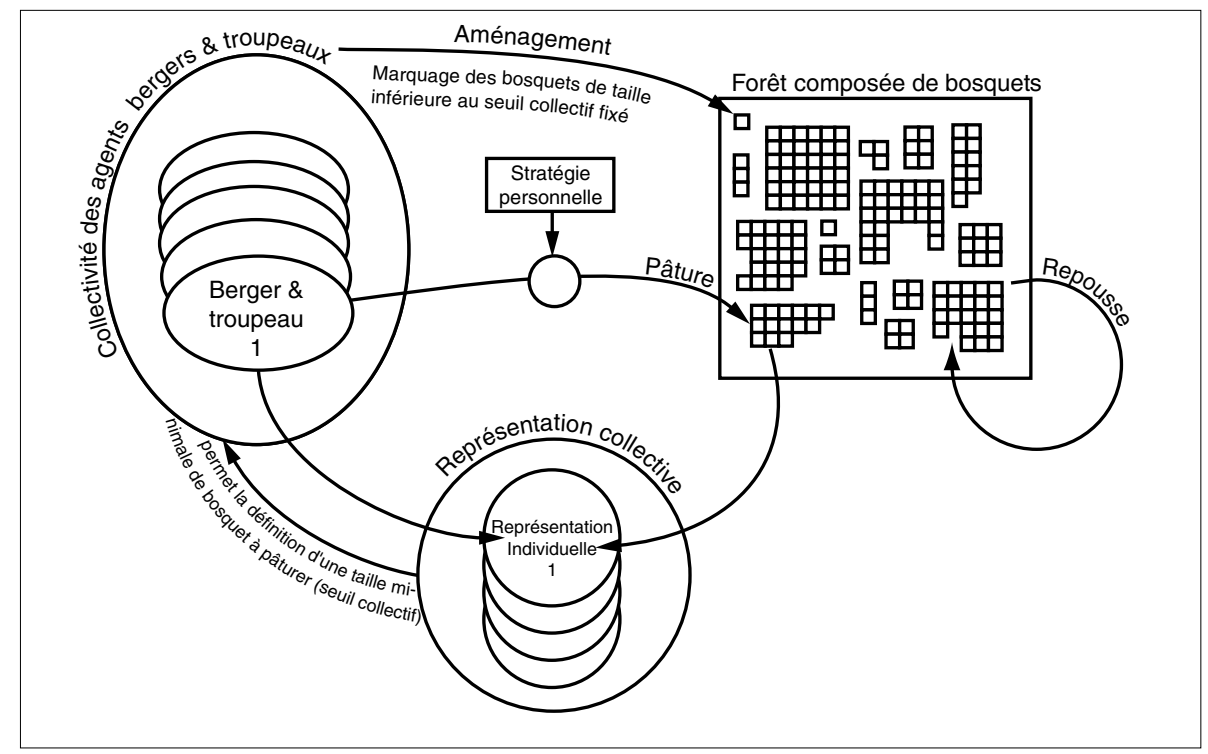

Fig. 2.- Architecture

du modèle.

Les agents «bergers et troupeaux » pâturent systématiquement les cellules sur lesquelles ils s'arrêtent, à moins que des règles collectives et leurs stratégies personnelles ne les en dissuadent. Nous reviendrons sur ces contraintes plus loin. Ces parcours conduisent à une dégradation de la forêt en savane. Parallèlement, la forêt repousse de manière naturelle selon une probabilité qui privilégie la diffusion à partir des lisières (la méthode utilisée pour calibrer cette repousse sera évoquée par la suite). À l'état initial, le taux de forestation de l'espace modélisé est de $27 \%$ avec une répartition en 11 bosquets de tailles diverses. À travers leurs pratiques spatiales, les agents «bergers et troupeaux» mémorisent les espaces parcourus. Un indicateur «mémoire trajet» propre à chacun cumule le nombre de cases de forêt parcourues sur une période de dix pas de temps pour constituer un «seuil individuel» issu du calcul (10 - mémoire trajet). Ainsi, le seuil individuel maximum est 10 si l'agent ne parcourt aucune cellule en forêt, il est nul si l'agent est resté pendant 10 pas de temps dans un espace forestier. Nous pouvons juger ici de la faible capacité de perception de l'espace dont nous avons muni l'agent «berger et troupeau». Il se déplace de manière aléatoire, toutefois sa perception dans un rayon d'une cellule lui permet, une fois une lisière forestière atteinte, de rester plus facilement dans le domaine forestier au lieu de divaguer au hasard. La seule mémoire qu'il possède est l'indicateur «mémoire trajet» incrémentant les cellules forestières effectivement parcourues. Ainsi la représentation de l'espace forestier pour l'agent «berger et troupeau» est très pauvre. Il ne se construit pas de carte mentale de son cheminement. Seule la quantité d'espace forestier parcouru fournit des éléments pour juger de l'état de la forêt en son entier. Nous pouvons considérer que l'agent procède par extrapolation pour fonder sa représentation globale de la forêt. Plus il parcourt d'espaces forestiers, plus sa représentation est celle d'une forêt abondante et vice-versa, quelle que soit la couverture forestière effectivement présente dans le modèle à ce moment de la simulation.

Ces représentations individuelles (appelées seuils individuels dans le modèle) sont confrontées les unes aux autres au sein du village tous les 10 pas de temps. Une représentation collective se construit, puis la mémoire individuelle est remise à zéro. Ici, cette construction collective est symbolisée par le calcul de la moyenne des indicateurs «mémoire trajet» de chaque agent «berger et troupeau». Ce nouvel indicateur qui établit un seuil limitant les parcours à venir est appelé «seuil collectif».

Une opération de gestion de l'espace va suivre, menée par la collectivité. Les bosquets de taille inférieure au seuil collectif feront l'objet d'un marquage. Dans ce dessin, les cases de la grille spatiale constituent également des agents «terrain» qui peuvent porter une occupation du sol en forêt ou savane. Le regroupement par contiguiité de cases en forêt ou des cases de forêt isolées sont reconnus comme des agents «bosquets». Cette possibilité de distinguer cases en 


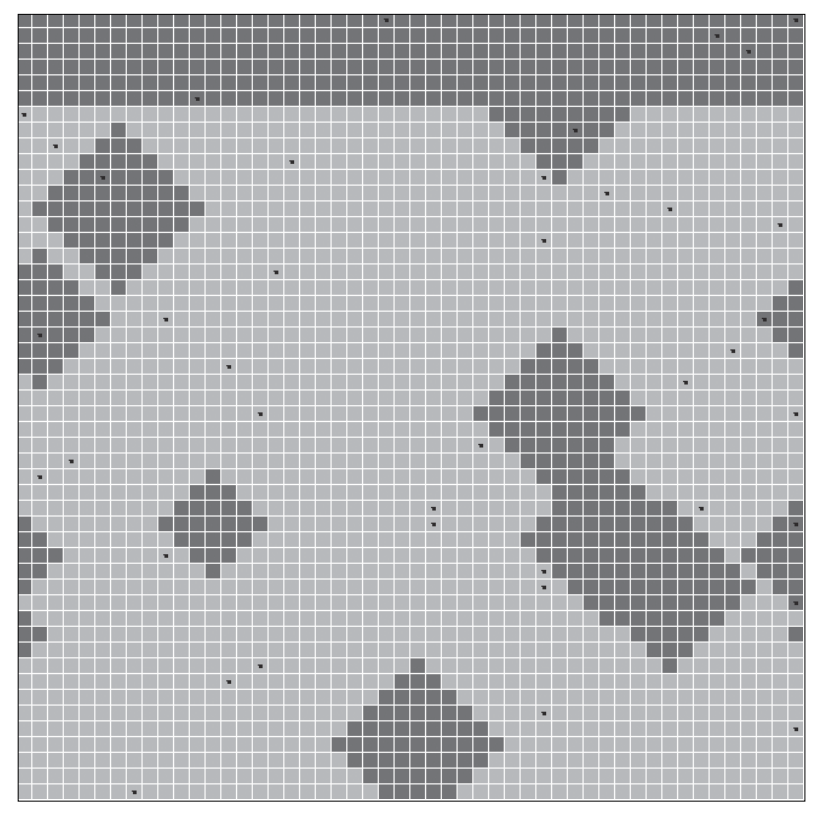

Fig. 3.-État initial.

La forêt est en gris foncé (686 cellules regroupées en 11 bosquets), la savane en gris clair. Les bergers accompagnant les troupeaux sont figurés par un point. Cet univers multi-agents est une grille fermée de 50 x 50 cases, chacune possédant 8 voisins.

forêt et agrégats de cases en forêt formant des bosquets est la manière utilisée dans le modèle pour appréhender des entités spatiales à méso-échelle. Les éléments : village, berger et troupeau, bosquet et terrain, sont identifiés par des variables et des processus qui leur procurent leur autonomie dans le programme (fig. 4$)^{5}$.

Chaque «bosquet» (regroupement de cases en forêt ou une case en forêt isolée) est identifié cycliquement par la collectivité et, selon sa taille (le nombre de cases qui le constitue) et le seuil collectif établi, un indicateur «drapeau» mentionne la décision de l'agent «village» le concernant. Si la taille du «bosquet» est supérieure au seuil collectif, il n'y a pas de pose de drapeau (valeur manquante pour l'indicateur), sinon l'indicateur porte le nombre de cellules

5. Les figures 4, 5, 6 et 7 ont été établies grâce à la formalisation UML (Unified Modelling Language) qui permet de s'affranchir des contraintes liées à l'environnement de programmation des systèmes multi-agents.

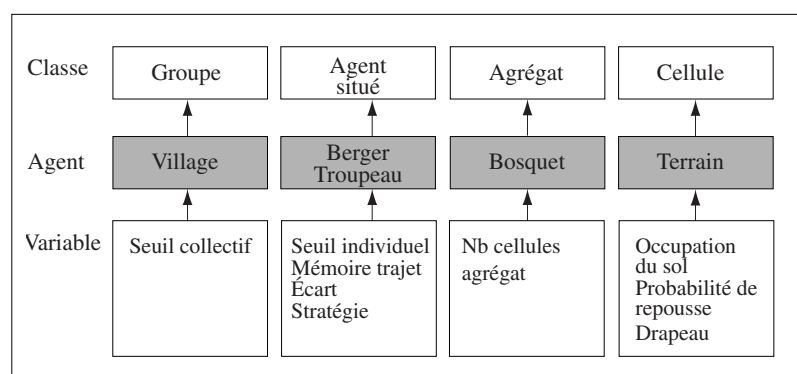

Fig. 4._Les composantes du modèle. Héritage de classe dans la plate-forme multi-agents.

en forêt qu'il comporte. L'agent «berger et troupeau» voulant alors entrer dans ce «bosquet» fera paitre ou non ses bêtes selon sa stratégie. En effet, plusieurs stratégies peuvent être données à l'agent «berger et troupeau». Le diagramme (fig. 5) décortique le comportement général d'un agent «berger et troupeau» en fonction de son voisinage (en forêt ou non) et de sa stratégie. Il peut avoir des pratiques individuelles conformes aux directives du groupe, cette stratégie

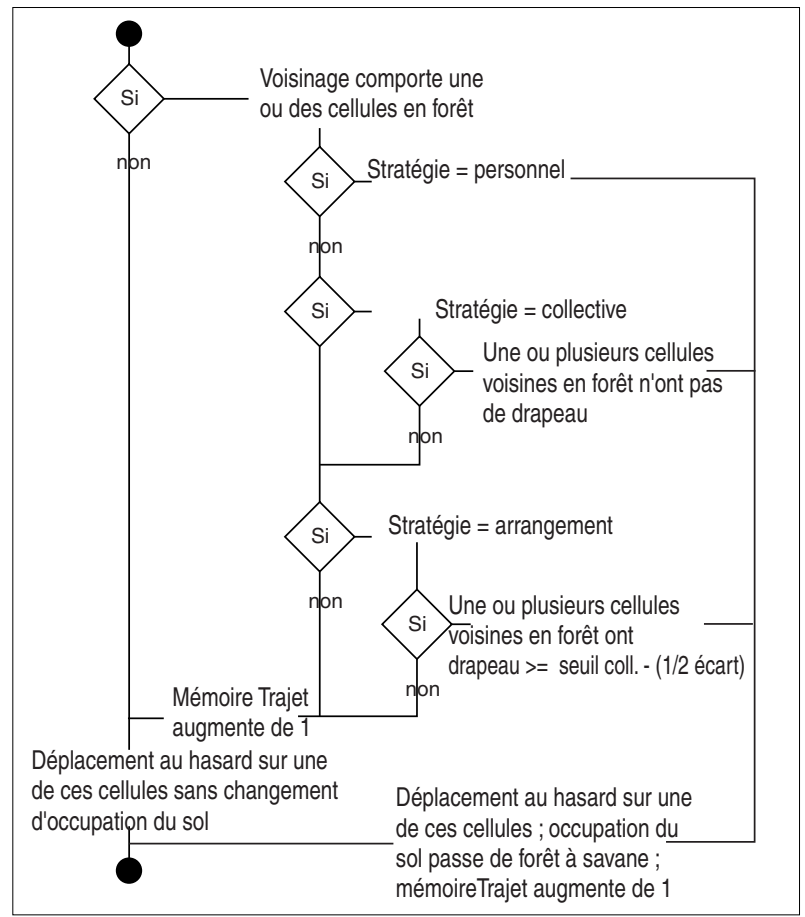

Fig. 5.- Comportement de l'agent «berger et troupeau» selon sa stratégie, son voisinage forestier et les décisions collectives. 


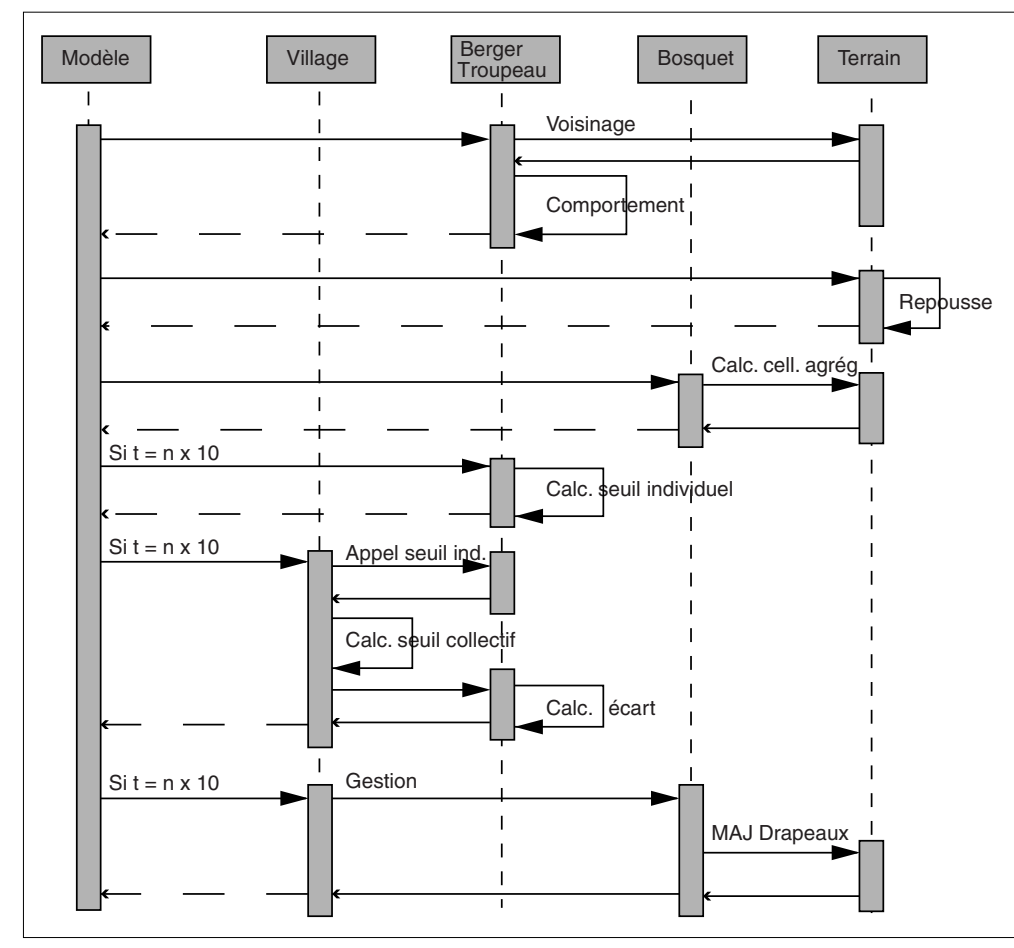

Fig. 6._-Diagramme de séquencement.

drapeaux d'interdiction que les agents «bergers et troupeaux» pourront franchir selon leur stratégie. Ensuite le cycle recommence.

\section{Quelques simulations}

Nous allons envisager des simulations successives où le jeu des représentations individuelles et collectives ira croissant. Deux simulations concernent la stratégie «personnelle» : la pratique des agents «bergers et troupeaux» influe sur celles des autres y compris s'il n'y a pas de contact direct entre eux. Cette modélisation des interactions se rapproche de ce que les économistes appellent des externalités. Dans un premier scénario, les représentations sont réduites à néant, le «berger» fait paître son troupeau dès qu'il rencontre la ressource. Ce scénario nous a permis de calibrer la repousse de la forêt qui est calculée pour chaque cellule à chaque pas de temps. C'est l'unique paramètre à étalonner dans ce modèle, les autres n'en ont pas fait l'objet (taille de la grille,

sera intitulée «collective». Il peut avoir des pratiques qui ne tiennent aucun compte de la règle collective et faire paître son troupeau systématiquement. Cette stratégie est dite «personnelle». Enfin, il peut déroger à la règle collective en adaptant ses pratiques, faisant un moyen terme entre son usage habituel et le seuil collectif. Cette stratégie est intitulée «arrangement». Dans le modèle, les stratégies des «bergers et troupeaux» sont établies en début de simulation et de manière permanente.

Le cadencement global de ce modèle est résumé par la figure 6. Nous y voyons l'enchaînement des différentes séquences annoncées plus haut : calcul du voisinage dans un rayon d'une cellule puis établissement du comportement des agents «bergers et troupeaux» selon ce voisinage et leur stratégie; repousse de la forêt en fonction d'un tirage aléatoire; calcul de la taille des nouveaux «bosquets». Les étapes suivantes ont une périodicité plus grande, tous les 10 pas de temps : calcul du seuil individuel à partir des trajets effectués; calcul du seuil collectif et des écarts entre collectif et chaque seuil individuel; puis mise à jour des surface en forêt, nombre d'agents «bergers et troupeaux»), reprenant certaines des valeurs déjà expérimentées (Bousquet, Gautier, 1999). La probabilité qu'une cellule passe de savane à forêt est le produit du nombre de ses cellules voisines en forêt par un paramètre constant à déterminer. Aussi, elle est nulle s'il n'y a aucune cellule en forêt parmi les huit voisins, et plus le voisinage est forestier, plus la probabilité de repousse est forte. Nous avons fait en sorte de choisir un paramètre susceptible d'illustrer la tragédie des communs où les biens collectifs sont exploités jusqu'à l'épuisement parce que les profits sont individualisés et les coûts partagés (Hardin, 1968). La valeur de ce paramètre a été fixée à 0,0025 et la forêt disparaît systématiquement à chaque simulation au bout de 120 à 250 pas de temps. La probabilité de repousse pour une cellule est au mieux égale à 0,02 quand huit cellules voisines sont en forêt. Bien sûr, des travaux ultérieurs intégrant notamment la sensibilité de la ressource aux interactions sociales (par exemple, Bousquet et al., 1996), ont montré les carences de cette hypothèse. Par ailleurs, la critique fondamentale de cette théorie concerne le fait que les ressources en biens communs ne 
Tabl. 1.-Résultats médians à l'issue de 10 simulations avec la stratégie «personnelle » et représentation individuelle.

\begin{tabular}{|lccccc|}
\hline & $\begin{array}{c}\text { Cases } \\
\text { en forêt }\end{array}$ & $\begin{array}{c}\text { Nombre } \\
\text { de bosquets }\end{array}$ & $\begin{array}{c}\text { Pâture moyenne } \\
\text { cumulée par berger }\end{array}$ & $\begin{array}{c}\text { Écart-type des pâtures } \\
\text { cumulées par berger }\end{array}$ & $\begin{array}{c}\text { Coefficient de variation des } \\
\text { pâtures cumulées par berger }\end{array}$ \\
\hline Valeur médiane & 169 & 15 & 26 & 21 & 0,8 \\
\hline
\end{tabular}

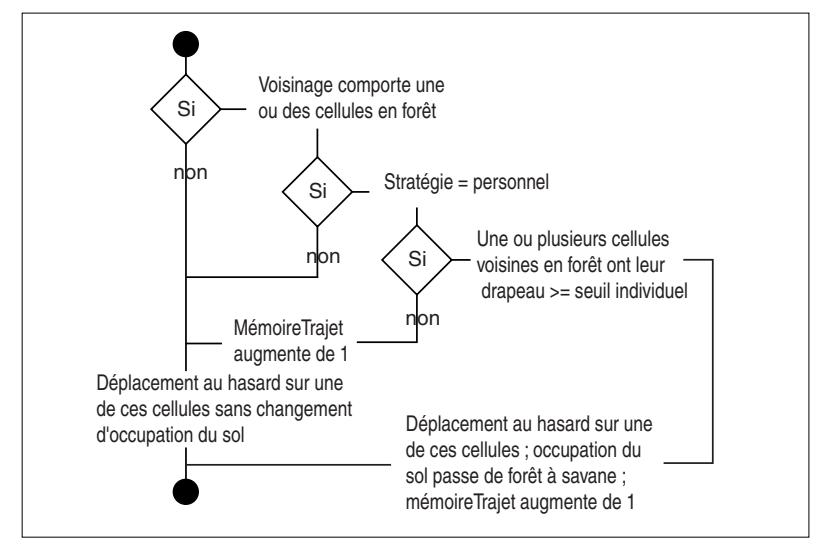

Fig. 7.- Comportement de l'agent «berger et troupeau» selon une variante de la stratégie personnelle.

sont pas forcément en accès libre. Les sociétés organisent des règles d'accès. Ce sera, entre autres, le sujet de nos simulations.

Un deuxième scénario inclut des représentations individuelles de la forêt au sein de cette stratégie «personnelle», toujours sans recours au groupe. C'est la propre représentation de la forêt par l'agent «berger et troupeau » dans le passé (10 pas de temps précédents) qui va conditionner la pâture. Si la forêt est dégradée, cet agent mémorise peu d'espaces boisés sur son trajet, son nouveau seuil individuel sera élevé 6 , il ne pourra faire pâturer les petits «bosquets» (fig. 7). Il est alors nécessaire que l'agent «berger et troupeau » connaisse la taille de l'agent «bosquet» dans lequel il est entré. Nous avons donc augmenté ses capacités de représentation pour cette simulation : l'évaluation de la taille du bosquet ne passe pas par le recours au groupe.

6. En effet, la « mémoire trajet » cumule le nombre de cases de forêt perçues sur une période de dix pas de temps pour constituer le seuil individuel issu du calcul (10 - mémoire trajet).

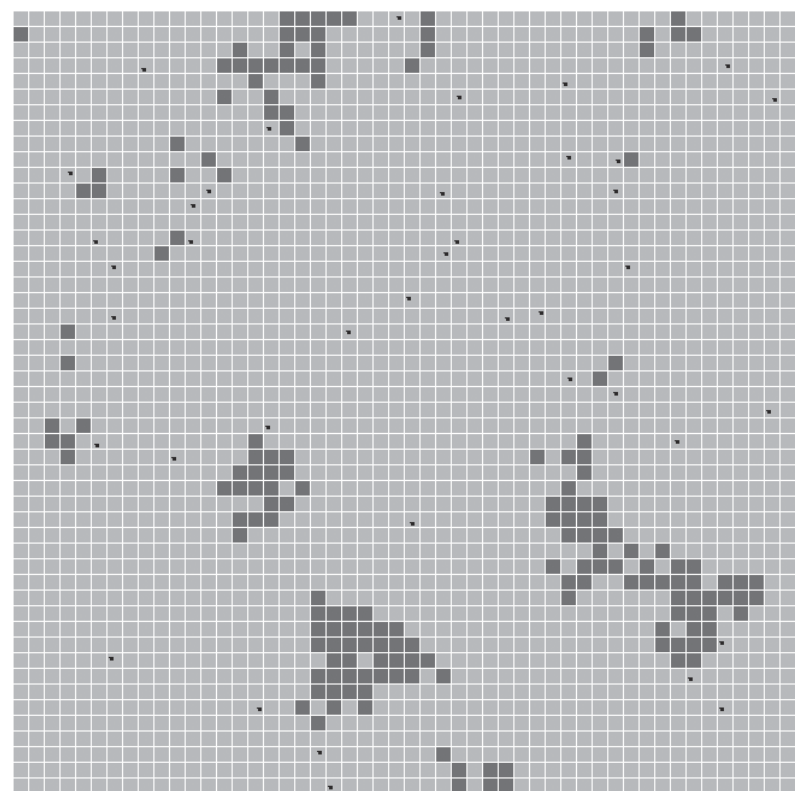

Fig. 8.- État forestier après 300 pas de temps pour la stratégie "personnelle» avec représentation individuelle.

Le résultat des simulations montre que la forêt se maintient à $25 \%$ de la forêt initiale en 15 «bosquets » (fig. 8 et tabl. 1). La pâture moyenne par agent «berger et troupeau» est faible (26 cases).

Les prochaines simulations introduisent la contrainte exercée par le groupe. La première stratégie est dite «collective». Là, les agents «bosquets» de taille inférieure au seuil collectif sont exclus de pâture par la «pose de drapeaux» d'interdiction et la stratégie des agents «bergers et troupeaux» suit strictement cette consigne. Les résultats des simulations montrent une régulation de la forêt à un niveau sensiblement plus élevé que dans le cas précédent (35\% de la forêt initiale au lieu de $25 \%$ ) mais elle est très fortement fragmentée en tout petits bosquets (fig. 9 et tabl. 2). La pâture moyenne cumulée est plus forte que précédemment, 32 contre 26 alors que les écarts entre «bergers et troupeaux» sont moins grands (coef. de variation $=0,6$ contre 0,8 ). 
Tabl. 2.-Résultats médians à l'issue de 10 simulations avec la stratégie «collective».

\begin{tabular}{|lccccc|}
\hline & $\begin{array}{c}\text { Cases } \\
\text { en forêt }\end{array}$ & $\begin{array}{c}\text { Nombre } \\
\text { de bosquets }\end{array}$ & $\begin{array}{c}\text { Pâture moyenne } \\
\text { cumulée par berger }\end{array}$ & $\begin{array}{c}\text { Écart-type des pâtures } \\
\text { cumulées par berger }\end{array}$ & $\begin{array}{c}\text { Coefficient de variation des } \\
\text { pâtures cumulées par berger }\end{array}$ \\
\hline Valeur médiane & 230 & 61 & 32 & 19 & 0,6 \\
\hline
\end{tabular}

Tabl. 3.- Résultats médians à l'issue de 10 simulations avec la stratégie "arrangement».

\begin{tabular}{|lccccc|}
\hline & $\begin{array}{c}\text { Cases } \\
\text { en forêt }\end{array}$ & $\begin{array}{c}\text { Nombre } \\
\text { de bosquets }\end{array}$ & $\begin{array}{c}\text { Pâture moyenne } \\
\text { cumulée par berger }\end{array}$ & $\begin{array}{c}\text { Écart-type des pâtures } \\
\text { cumulées par berger }\end{array}$ & $\begin{array}{c}\text { Coefficient de variation des } \\
\text { pâtures cumulées par berger }\end{array}$ \\
\hline Valeur médiane & 233 & 63 & 32 & 21 & 0,7 \\
\hline
\end{tabular}

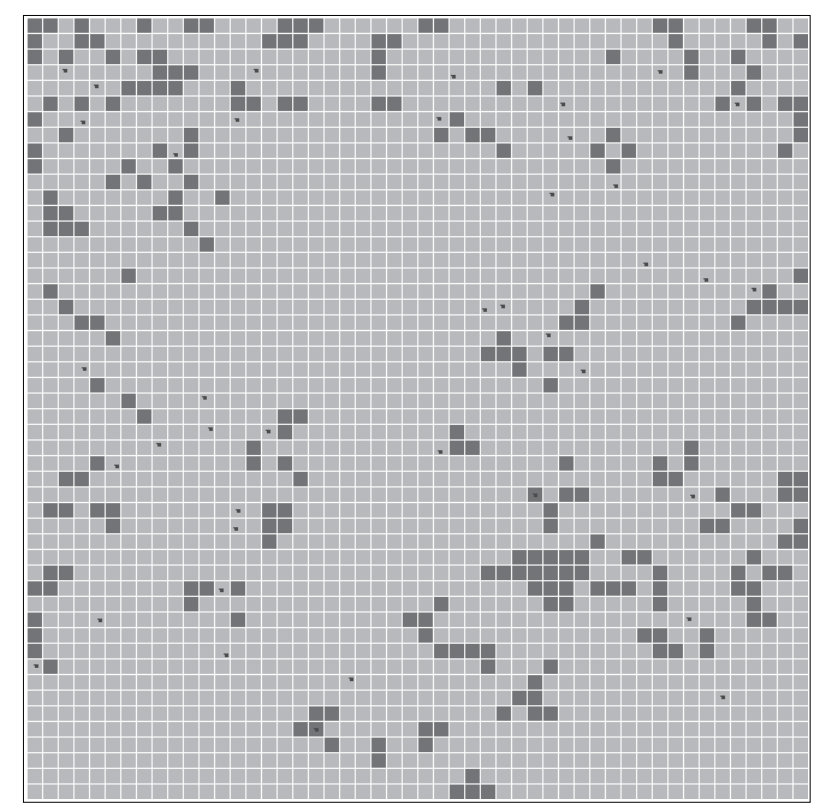

Fig. 9.—État forestier après 300 pas de temps pour la stratégie "collective».

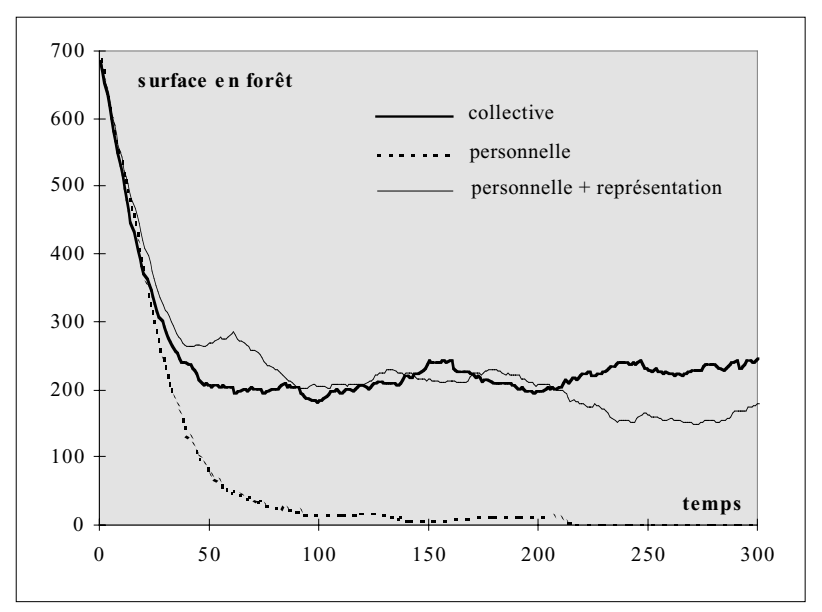

À partir de cette stratégie «collective», la stratégie «arrangement» peut apparaître comme une dérogation que s'accorde l'agent «berger et troupeau». Coupant la poire en deux, il s'autorise la pâture de «bosquets» de la taille fixée collectivement diminuée de la moitié de l'écart à son propre seuil (cf. fig. 7). Les simulations de ce type ont des résultats très proches de la stratégie «collective» à quelques nuances près (tabl. 3).

\section{Interprétation}

Dans le premier scénario qui fait suite au calibrage de la repousse (stratégie «personnelle» augmentée d'une représentation individuelle de la forêt), l'agent «berger et troupeau» voit dans ses nouvelles représentations et subit dans sa consommation les conséquences de sa propre consommation par le passé ou celle des agents concurrents. Nous pourrions dire qu'il refrène sa consommation et considérer que c'est une «prise de conscience» par l'agent «berger et troupeau» de la finitude de la ressource qui est modélisée. L'intégration dans le modèle d'une représentation et d'une gestion individuelles du couple forêt-pâture aboutit à une régulation de la ressource (fig. 10). Ainsi, sans recours au groupe, ce modèle fournit une alternative à la tragédie des communs. L'agent «berger et troupeau » gère sa préemption en fonction de ses représentations dans le local.

Fig. 10.-Évolution de la superficie boisée sur 300 pas de temps pour les stratégies "personnelle», "personnelle avec représentation individuelle», et «collective». 
Cependant, compte tenu du fort écart-type (tabl. 1), ces chiffres mettent en évidence une grande disparité des occasions spatiales individuelles (rencontre aléatoire d'un «bosquet» de taille importante, ce qui entraînera une représentation exubérante de la forêt et une propension à faire pâturer sans retenue, et vice versa), ou économique (concurrence avec d'autres agents, à savoir le fait d'être arrivé avant les autres sur le lieu de pâture). S'il y a de grandes disparités dans les consommations des agents «bergers et troupeaux», la ressource en fin de la simulation est constituée d'un nombre réduit de bosquets à l'opposé de la simulation centrée sur la stratégie «collective».

Avec plus de 60 «bosquets» en fin de simulation pour 11 à l'instant initial, le scénario à stratégie «collective» provoque un véritable émiettement de la forêt. Ceci ne fait que traduire de manière spatiale la méthode utilisée par le groupe pour exercer une contrainte sur les agents «bergers et troupeaux». Puisque l'interdiction porte sur une taille minimum et qu'elle est valable pour tous, il y a une réification de la règle collective, chose impossible dans le cas précédent où chaque agent «berger et troupeau» construit son propre seuil. Si une contrainte de ce type exercée par le groupe ne correspond guère à une situation réelle (les espaces boisés protégés sont souvent de grande taille et peu nombreux), elle apparait ici socialement efficace : les troupeaux pâturent plus et les écarts sont moins grands. Il y a une apparente normalisation effectuée par le groupe qui réduit les inégalités entre agents «bergers et troupeaux». Nous pouvons imaginer que c'est l'atomisation de la forêt en petits «bosquets» qui multiplie les opportunités spatiales pour chaque agent «berger et troupeau» d'atteindre des parcelles en forêt. Nous verrons par la suite que cette explication spatialiste est insuffisante.

Il y a une régulation de la forêt au cours de la simulation malgré la connaissance imparfaite de l'environnement forestier par les agents ou le groupe. En comparant le seuil collectif, c'est-à-dire la représentation collective, à la réalité des faits dans cet univers multi-agents, nous pouvons juger du décalage car cette «moyenne» des représentations individuelles traduit de manière comptable, c'est-à-dire non spatialisée, l'ampleur de la forêt. Le seuil collectif est égal à 5 en fin de simulation. Il suppose que l'agent «berger et troupeau » moyen estime que la moitié de l'espace est boisée. Or il n'y a que $9 \%$ de forêts. Les sources d'écart sont multiples, entre réalité virtuelle et représentation collective, comme nous venons de le voir, mais également entre représentations collective et individuelle ou entre représentation individuelle et univers artificiel. En effet, l'agent «berger et troupeau» n'a pas un parcours totalement erratique car il privilégie le maintien en forêt, et une même case en forêt peut être comptabilisée plusieurs fois dans la période de référence; ceci implique une survalorisation automatique du couvert forestier. De plus, les représentations individuelles ne reflètent pas la réalité objective de cet univers multi-agents puisque l'agent n'a évolué que sur une petite part de l'espace disponible et que son expérience personnelle et passée ne présage en rien de l'état actuel de la forêt. Or, de ces représentations individuelles émergent une représentation collective qui constitue une règle commune, ainsi qu'un accès pour chaque «berger et troupeau» aux représentations de tous. Ainsi, sur la base de représentations anciennes, les directives de gestion vont s'appliquer pour les dix pas de temps à venir alors même que la pâture se poursuit et que la forêt se régénère. D'une part, l'augmentation naturelle de la taille des bosquets peut rendre caduque l'interdiction de pâture. D'autre part, des «bosquets» à peine trop grands pour faire l'objet d'une interdiction peuvent être totalement déboisés avant la prochaine décision collective puisque c'est l'agent «village» qui détient la responsabilité de l'interdiction et non l'agent «berger et troupeau».

Cela soulève une question intéressante concernant l'effet de la privation ou non de responsabilité individuelle dans le cadre d'une gestion durable. Cette option a été simulée pour évaluer son effet sur le couvert forestier : l'agent «berger et troupeau» exerçant un autocontrôle en comparant la taille du «bosquet» au seuil collectif au moment où il veut le faire pâturer par ses bêtes. Il y a un effet immédiat puisqu'une partie de l'inertie disparaît, celle liée aux intervalles de décision, mais les résultats sur un nombre limité de simulations n'ont pas montré de grandes modifications par rapport à la stratégie «collective». Cela ne répond pas cependant à la redoutable question de savoir ce qu'il subsiste d'un niveau de responsabilité collective lors $\mathrm{du}$ «passage» à la raison individuelle?

Dans la stratégie «arrangement», nous avons constaté que le nombre de cases en forêt était très fluctuant d'une simulation à l'autre, traduisant explicitement sur le plan comptable des oscillations entre stratégie «personnelle» et 
stratégie «collective». Il est aussi intéressant de noter que la modélisation d'une adaptation individuelle de la règle collective introduit une augmentation des écarts entre agents concernant le cumul d'espaces pâturés. On constate également une fragmentation légèrement supérieure de la forêt et ce résultat apporte un bémol à l'hypothèse lancée lors de la simulation avec stratégie «collective», qui consistait à faire de l'atomisation de la forêt en petits bosquets le déterminant d'une relative équité de pâture. À la vue de ces derniers résultats, il semble que le respect de la règle collective favorise, à terme, la réduction des écarts initiaux, qui sont liés à la position relative de chacun (par rapport à la forêt et aux autres agents «bergers et troupeaux»), avantageuse ou pas.

Toutes ces connaissances imparfaites, que nous avons modélisées en introduisant un décalage entre perception et réalité de l'univers multi-agents, participent à l'enrichissement de la dynamique et à la régulation de la ressource. Ainsi, si la stratégie «personnelle» réfère à la notion d'externalité, nous pouvons dire que la stratégie «collective» réfère à la théorie des conventions, la «collectivisation» des représentations au sein de la société des agents servant ici d'aiguillon au triptyque individu, espace et société. Sur le plan de la modélisation, c'est un médiateur et un catalyseur entre ces trois pôles.

\section{Conclusion}

La grande simplicité du modèle en termes de construction et de paramètres, la rusticité de la construction de la représentation collective (une moyenne arithmétique) mettent en avant la robustesse du modèle dynamique établi entre espace et représentations individuelles et collectives. Nous sommes portés à croire que cette robustesse a pour origine la définition de l'espace géographique évoquée plus haut, polie par l'usage des géographes et par leur recherche, construction incluant des représentations en interaction avec l'espace et la société. Dans l'univers de la programmation SMA, cette modélisation est une des rares boucles individus-sociétés où la boucle de retour de la société vers les individus passe par une contrainte sur la relation à l'espace, appréhendé à deux niveaux d'organisation, la case en forêt et le bosquet. Inversement, de la relation des individus à l'espace se construit une représentation collective.
La modélisation du jeu des représentations spatiales que se forgent les agents «bergers et troupeaux» au cours de leurs actions se révèle intéressante dans le cadre des SMA et pour l'approche de l'espace géographique (Bonnefoy et al., 2000). Notamment, cette modélisation montre la forte capacité de la collectivité à produire des formes spatiales dès qu'elle émet une norme susceptible de régler des pratiques individuelles. Nous en avons des exemples évidents dans notre expérience de géographe, avec la réification du modèle des lieux centraux par l'entremise de la construction de grandes pénétrantes de massification des flux (Bonnefoy, 1998). Enfin, elle montre également le poids des formes spatiales sur les pratiques individuelles, aspect que nous pourrions exploiter plus avant en faisant varier la structuration de l'espace initial.

Finalement, cette modélisation nous renvoie à notre discipline : structures spatiales et représentations peuvent-elles s'unir dans une même géographie? Une structure spatiale constitue-t-elle la résultante de la pratique du plus grand nombre en même temps qu'elle est la mise en acte d'une règle collective, elle-même pouvant être le fruit d'une interaction entre espace et individus? Quel est le poids de chacune de ces propositions dans l'émergence d'une forme particulière?

Ce type de modélisation permet de mieux appréhender (visualiser et quantifier) les effets d'émergence à mésoéchelle de pratiques individuelles réitérées. Cependant, tous ces aspects ne peuvent être éclaircis sur cet exemple, même s'ils constituent un des moteurs de notre recherche à venir. Une telle démarche est en soi une réponse originale pour une approche des phénomènes complexes. Pour le géographe, c'est une occasion supplémentaire de mettre en espace une approche comportementale, distribuée au niveau des individus, par la définition des agents et de leurs règles d'interaction, non par des formules lourdement paramétrées des systèmes dynamiques qui prennent plus en compte les flux entrants et sortants que l'aspect comportemental des interactions.

Enfin, c'est un moyen efficace pour un chercheur de construire un dispositif expérimental, c'est-à-dire de mettre en scène ses théories ou modèles spatiaux, ses hypothèses, de simuler et de confronter les faits issus de processus au sein de cet univers multi-agents à une «réalité» observée. Car c'est un des intérêts de la modélisation 
par les systèmes multi-agents que d'avoir un grand effet heuristique en poussant le simulateur à s'interroger plus avant sur ses propres conceptions du monde à travers sa discipline et ses partis pris.

\section{Références}

Allen P.M., SANGLier M. (1981). «Urban evolution, selforganization and decision-making $»$. Environnement and Planning, $\mathrm{n}^{\circ} 13$, p. 167-183.

Atlan H. (1981). «L'émergence du nouveau et du sens», in Dumouchel P., Dupuy J.-P. dir., L'Auto-organisation. De la physique au politique. Colloque de Cerisy. Paris : Le Seuil, p. 115-129.

BONNEFOY J.-L. (1998). «Circularité entre modèles spatiaux et décisions spatiales ». Colloque Géopoint. Avignon : Groupe Dupont, p. 96-99.

Bonnefoy J.-L., Le Page Chr., Rouchier J., Bousquet Fr. (2000). «Modelling spatial practices and social representations of space using multi-agent systems ». International Conference on Computer Simulations and the Social Sciences, Université Panthéon-Assas Paris II, 18-20 septembre.

Bousquet Fr., Barreteau O., Mullon C., Weber J. (1999). «Modélisation d'accompagnement : systèmes multiagents et gestion des ressources renouvelables». Colloque Quel environnement, maîtrise du long terme et démocratie. Paris : Hermès, à paraître.

Bousquet Fr., Duthoit Y., Proton H., Le Page J., Weber Chr. (1996). «Tragedy of the commons, game theory and spatial simulation of complex systems ». Colloque Écologie, société, économie : quels enjeux pour le développement durable. Université de Versailles-Saint-Quentin- en-Yvelines, 8 p, 23-25 mai.

Bousquet Fr., Gautier D. (1999). «Comparaison de deux approches de modélisation des dynamiques spatiales par simulation multi-agents : approches spatiales et acteurs».
Revue Européenne de Géographie CYBERGEO, article numéro 89.

Brunet R., Ferras R., Théry H. (1992). Les Mots de la géographie. Dictionnaire critique. Paris/Montpellier : RECLUS/ La Documentation française.

Bura S., Guérin-Pace Fr., Mathian H., Pumain D., Sanders L. (1996). "Multi-agents systems and the dynamics of a settlement system». Geographical Analysis, 2, 161-178.

Cattan N., Grasland Cl. (1994). Dynamiques migratoires et recompositions territoriales en Tchécoslovaquie de 1960 à 1992. Paris : CNRS.

Conway J. (1970). «Mathematical games». Scientific American, october, p. 120-127.

Drogoul A., Corbara B., Fresneau D. (1993). «Manta : new experimental results on the emergence of (artificial) ant societies». Simulating Societies Symposium. Sienne : C. Castelfranchi.

FERBER J. (1995). Les Systèmes multi-agents. Vers une intelligence collective. Paris : InterÉditions (2e éd. 1997).

FrANKHAUSER P. (1994). La Fractalité des structures urbaines. Paris : Économica, coll. «Villes».

HARDIN G. (1968). «The tragedy of the commons». Science, $n^{\circ} 162$, p. 1243-1248.

HAYNES K.E., FotheRINGHAM A.S. (1988). Gravity and Spatial Interaction Models. Beverly Hills : Sage, «Scientific Geography».

Heudin J.-C. (1994). La Vie artificielle. Paris : Hermès.

LANSING J.S., KREMER J.N. (1994). «Emergent properties of the Balinese water temple networks : coadaptation on a rugged fitness landscape». Artificial Life III, C.G. Langton Addison Wesley ed.

NeUman J. von (1966). Theory of self Reproducing Automata. Urbana (Ill.) : University of Illinois Press.

Pumain D., SAInT-Julien Th., SANDERs L. (1989). Villes et auto-organisation. Paris : Économica.

SANDERS L. (1992). Systèmes de villes et synergétique. Paris : Anthropos, coll. «Villes».

WiLSON A. (1970). Entropy in Urban and Regional Modelling. Londres : Pion. 